\title{
mTOR in inflammatory skin diseases: Review of the literature and our experience
}

\author{
Cacciapuoti $S^{1}$, Marasca $C^{1}$, Luciano $M^{2}$, Masarà $A^{1}$, Donnarumma $M^{1}$, Balato $A^{1}$ and Fabbrocini $G^{1}$ \\ ${ }^{1}$ Department of Clinical Medicine and Surgery, Section of Dermatology, University of Naples Federico II, Italy \\ ${ }^{2}$ Department of Public Health, University of Naples Federico II, Italy
}

\section{Introduction}

The mechanistic Target of Rapamycin (mTOR) is a serine/ threonine kinase, involved in a wide range of biological processes, including cellular metabolism, growth, proliferation, and survival. It was discovered in the early 1990s as the target of the anti-fungal drug rapamycin [1].

mTOR responds to numerous environmental signals and it has been recently found that it plays also a role in lipogenesis and gluconeogenesis [1].

MTOR kinase acts in two functionally distinct complexes, mTOR complex 1 (mTORC1) and two (mTORC2), whose activities and substrate specificities are regulated by complex co-factors [2].

The mTORC1 protein complex consists of multiple components such as the serine/threonine kinase $\mathrm{mTOR}$ and the regulatoryassociated protein of mTOR (Raptor). Three highly researched upstream modulators of mTOR are the phosphatidylinositol 3-kinases (PI3Ks), protein kinase B (Akt). PI3K, and tumor necrosis factor alpha (TNFa) regulates Akt. Increased expression of PI3K, Akt, and TNFa amplifies mTORC1 activity as well (Figure 1) [3,4].

Extensive researches over the past two decades have established the involvement of mTOR in the progression of several diseases, such as cancer, diabetes and numerous inflammatory skin diseases.

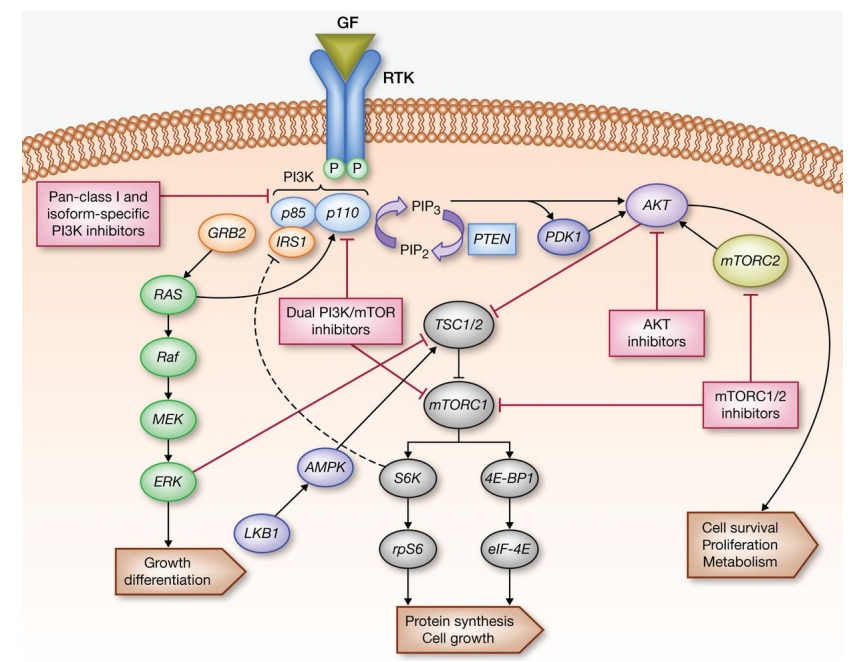

Figure 1. Schematic representation of the PI3K/AKT1/MTOR Signaling. (Adapted from Picking the Point of Inhibition: A Comparative Review of PI3K/AKT/mTOR Pathway Inhibitors, Rodrigo Dienstmann, Jordi Rodon, Violeta Serra and Josep Tabernero)
In this review, we report recent advances regarding mTOR role in some invalidating inflammatory skin diseases. We also discuss the current and future prospects for therapeutic targeting mTOR in the clinical practice.

\section{Methods}

For this review, we searched published data concerning mTOR in skin diseases in the following databases: PubMed, Embase, Cochrane Library, Scopus. Herein we have described the role of mTOR in Psoriasis, Atopic dermatitis, Pemphigus vulgaris, Acne, Hidradenitis suppurativa.

This article is based on previously conducted studies.

\section{mTOR in inflammatory skin diseases}

It has been shown that the mTOR pathway is involved in the pathogenesis of several dermatological conditions such as epithelial tumors, melanoma, mycosis fungoides, sclerosis tuberose, psoriasis, acne, wound healing and recently hidradenitis suppurativa [5]. Regarding the immune system, mTOR showed to have an important regulating role. Indeed, the rapamycin inhibits mTOR suppressing the ability of T lymphocytes to respond to IL-2 and thus to proliferate in response to antigenic stimulation. Therefore, mTOR reduces $\mathrm{T}$ regulatory lymphocytes and appears influence the function of dendritic cells. In addition, according to Delgoffe et al. mTOR may inhibit the expression of FOXP3 (forkhead box P3), transcription factor for T regulatory lymphocytes, modulating $\mathrm{T}$ helper cell differentiation in Th1, Th17 and Th2 [6]. Moreover, protein kinase B (Akt) is able to activate $\mathrm{T}$ Helper lymphocytes and to limit the differentiation of regulatory $\mathrm{T}$ cells, playing a key role in immune tolerance from self-antigens and antigens from the external environment [7]. About the carcinogenesis, it has been described that mTOR pathway is associated with skin melanomas and others skin cancers caused by UVA and UVB exposure [8]. In particular, mTOR has a pivotal role in melanomas. In a recent survey 76 out of 107 melanomas had moderate to severe increase of S6K1 phosphorylation, while only $3 / 67$ benign nevi showed moderate

${ }^{\star}$ Correspondence to: Sara Cacciapuoti, Department of Clinical Medicine and Surgery, Section of Dermatology and Venereology, University of Naples Federico II, Via Pansini 5, Naples, Italy, Tel: +390817462435; Fax: +390817462412; E-mail: sara.cacciapuoti@libero.it

Key words: $m$ TOR, skin inflammatory disease

Received: January 24, 2019; Accepted: February 13, 2019; Published: February 18,2019 
increase of this phosphorylation [9]. All these evidences supports the idea that mTOR pathway plays a prominent role in inflammatory skin diseases.

\section{mTOR in psoriasis}

Healthy epidermis homeostasis is expressed by keratinocyte stem cells asymmetrically division, with keratinocyte leaving the basal layer and developing into the spinous, granular and corneal layers, characterized by ordered expression of keratins and other markers such as involucrin, loricrin, filaggrin or transglutaminase [10]. The balance between keratinocyte proliferation and differentiation is dysregulated in certain skin diseases such as psoriasis [11]. Psoriasis is a chronic inflammatory disease of the skin involving a complex interplay of genetic, environmental, and immunologic factors [12]. It is a common disease (prevalence estimated up to $3 \%$ of general population), clinically characterized by well-demarcated, red, scaly plaques about the size of a palm. Epidermal hyper-proliferation, inflammation and angioneogenesis are usually found in psoriasis. Skin biopsy revealed an irregular stratum corneum with parakeratosis, epidermal thickening with acanthosis and absence of the granular layer. This is caused by hyperproliferation of keratinocytes that are unable to properly initiate the epidermal differentiation program [13].

Psoriasis is an immunological disease, characterized by an intimate interplay between the innate and the adaptive immune systems. The central axes of psoriatic inflammation comprise Th17/ Th2 2 cells, the nuclear factor-kB, interferon-g, and interleukin (IL)-23 signaling pathways as well as antigen presentation [14].

mTOR plays a crucial role in skin homeostasis and morphogenesis, especially in the regulation of keratinocyte differentiation and epidermal stratification [15]. Punch biopsies from patients with severe plaque psoriasis showed elevated levels of phosphorylated mTOR (P-mTOR) in the basal layer as well as its downstream target S6K1 in suprabasal layers. This suggests that mTORC1 plays a role in the pathogenesis of psoriasis $[16,17]$. In addition, the PI3-K/mTOR pathway can play a role in Th1-Th2-Th17 imbalance in the pathogenesis of psoriasis [18]. mTORC1 regulates the activation of downstream targets $\mathrm{p} 70 \mathrm{~S} 6 \mathrm{~K}$ and $4 \mathrm{E}-\mathrm{BP} 1$ resulting in protein synthesis and cell growth [19]. In fact, the key pathologic outcome in psoriasis is the 'uncontrolled proliferation' of keratinocytes [20]. Buerger et al. found that under healthy conditions mTOR signaling was deactivated when keratinocytes switch from proliferation to terminal differentiation. Inflammatory cytokines (IL-1 $\beta$, IL-17A, TNF- $\alpha$ ) indeed, induced aberrant mTOR activity which led to enhanced proliferation and reduced differentiation. Conversely, regular differentiation could be restored if mTORC1 signaling is blocked. When mTORC1 signaling is inhibited by Rapamycin treatment in cellular differentiation models, the expression of differentiation markers like involucrin was increased on the protein level. Thus, inactivation of mTORC1 signaling is a prerequisite for the progression of differentiation. Furthermore, it was found a hyper-activation of different components of mTOR signaling in psoriatic skin with several localizations over the lesional skin epidermis but especially intense phosphorylation in the basal layer and increased activity in healthy skin. IL-1 $\beta$, IL-17A and TNFastrongly activated the mTOR kinase, PRAS40 and the downstream targets of mTOR activity. The effect of psoriatic cytokines on mTORC1 prevents proper epidermal stratification. In summary, these results not only identify mTORC1 as an important signal integrator pivotal for the cells fate to either proliferate or differentiate, but emphasize the role of inflammation dependent mTOR activation as a psoriatic pathogenesis [11]. Mitra et al. have studied the potential therapeutic role of 1a; 25-dihydroxyvitamin D3-3-bromoacetate (BE), a Vit D analog, finding that $\mathrm{BE}$ is significantly more potent than an equivalent concentration of Vit-D in inhibiting growth and survival of human keratinocytes [21]. The antimitotic effect of BE is due to the inhibition of phosphorylation of serine/threonine protein kinase (AKT) and its downstream target, mTOR. In the reconstructed human epidermal model (RHE), BE reversed IL-22-induced psoriasiform changes more effectively than Vit-D. Moreover, the number of cells returning in the granular layer of the epidermis with $\mathrm{BE}$ treatment were more than that observed with Vit-D treatment, confirming regulation of AKT/mTOR pathway behind its antiproliferative effect. The mechanism for the antimitotic effect of Vit-D is quite complex and it may differ according to the anatomical site and kind of cells; particularly, in psoriatic keratinocytes it induces apoptosis [22,23]. This suggests that both BE and Vit-D may be potential therapeutic agents for psoriasis through inhibition of Akt/mTOR inhibition. These cell culture findings warrant further examination in animal and human tissue to assess the clinical significance [24,25].

\section{mTOR in atopic dermatitis}

Atopic dermatitis (AD) is a common chronic inflammatory skin disease, characterized by pruritic and eczematous skin lesion, usually associated with personal or family history of allergic diseases, including $\mathrm{AD}$, asthma, and allergic rhinitis [26]. $\mathrm{AD}$ is characterized microscopically by spongiotic dermatitis with moderate dermal immune cell infiltrates. The skin inflammation is thought to be due to a complicated interaction between activated Th2 lymphocytes, eosinophils and mast cells [27]. Although immunological or hereditary abnormalities in $\mathrm{AD}$ have become clear in recent years, topical glucocorticoid application is still the most effective therapy and it is the standard treatment in many guidelines, but there is a need for more effective alternative therapies without the negative side effects of glucocorticoids [28]. Very few data analyse the role of mTOR in the pathogenesis of atopic dermatitis, and most of these study had investigated the therapeutic efficacy of different molecules able to inhibit mTOR, improving the clinical outcome of AD patients.

In $\mathrm{AD}$, basophils and mast cells mediate chronic allergic inflammation, which is dependent on the high affinity immunoglobulin E (IgE) receptor type I (FcERI). In the response to IgE, these cells rapidly release intracellular mediators like interleukins, leukotrienes and histamine. Major FceRI downstream pathways include the mitogen-activated protein kinase (MAPK)/extracellular signal-related kinase (ERK) pathway and the phosphoinositide 3-kinase (PI3-kinase)/ Akt pathway [29]. Luskova et al. have highlighted that mTOR and p70 ribosomal protein S6 kinase (p70S6K), PI3- kinase downstream signaling molecules, are considered potential therapeutic targets for diseases associated with activated basophils and mast cells or abnormal mast cell growth, like AD [30]. More recently, Yang et al. evaluated the effect of topical rapamycin in a murine model of $\mathrm{AD}$, developed by repeated application of Dermatophagoides farina body (Dfb) extract ointment on skin of NC/ Nga mice. Rapamycin, a well-known secondgeneration immunosuppressive agent, binds to the immunophilin FK506-binding protein-12, inhibits the activation of mTOR, preventing cell cycle progression from $\mathrm{G} 1$ to $\mathrm{S}$ phase, leading to suppression of $\mathrm{T}$ lymphocyte activation and antibody production. Moreover, the selective inhibition of mTORC2 leads to a reduction in mast cell proliferation associated with inflammation. Rapamycin induced a clinical improvement of skin lesions in treated mice, a reduction in scratching, the inhibition of nerve growth factor (NGF) expression in lesional skin (which is known to reflect the severity of itch and eruption), a reduction 
in the epidermal thickness and in the number of infiltrating cells in the dermis, compared with mice in non-treatment or vehicle group [31]. These results indicated that treatment with rapamycin improved itch and scratching in Dfb-induced atopic dermatitis mice [32]. OsadaOka et al.evaluated the therapeutic efficacy of a methanol extract of red ginseng (RGE) on AD skin through the inhibition ofmTOR/ p7056K signaling in human basophils and keratinocytes, examining the relationship between the anti-allergic mechanisms of RGE and the mTOR/p70S6K signal pathway in AD mice [33]. Many previous studies have shown that red ginseng inhibits PI3-kinase, Akt, and MAP kinase signaling pathways [34]. This one instead revealed that, RGE, inhibited p70S6K phosphorylation induced by FceRIa in basophils and IFN-g in keratinocytes, identifying RGE as a potential therapeutic agent against inflammatory responses involving activation of p70S6K signaling in $\mathrm{AD}$ [35]. The pathogenesis of $\mathrm{AD}$ is immunologically complex, Thelper type 2 (Th2) cytokines are important for the development of $\mathrm{AD}$, while the $\mathrm{T}$ helper type 1 (Th1) cytokine IFN- $\gamma$ in the maintenance of the chronic stage of $\mathrm{AD}$ [36]. Regulatory T cells (Tregs) have a curative role in the pathogenesis of $\mathrm{AD}$ and the severity of the disease is negatively correlated with the percentage of Tregs [37]. Being the foxp $3^{+}$Tregs essential in reducing skin inflammation, the expansion of this cell population can be used as a treatment in the AD. Min-Jung Bae et al. studied whether PG102, a water-soluble extract from an edible fruit, A. arguta, that according to the theory of Korean traditional medicine, reduces the unregulated overflow of energy that characterizes allergic reaction, may improve, if orally administered, the allergic symptoms of $\mathrm{AD}$ induced by the Dermatophagoides farinae extracts (DfE), as well as PG102 has any effect on the Treg generation. PG102 decreased the clinical score, resulting in the prevention of inflammatory cytokine infiltration in the dermis. Furthermore, oral administration of PG102 induced the generation of CD4pFoxp3p Tregs and suppressed the activation of Th1, Th2 and Th17 cells by DfE in the inflammatory region. These in vitro and in vivo data clearly indicate that PG102 might act as an effective immunosuppress or for AD [38].

New biological agents including anti-IgE (omalizumab), antiIL-5 (mepolizumab), anti-CD11a (efalizumab), have recently been introduced for $\mathrm{AD}$ treatment. Hamilton et al. showed that anti-IL-4R (dupilumab) is able to suppress gene expression related to activation of $\mathrm{T}$ cells, eosinophils, inflammatory pathways and TH2-inducing chemokines [39]. A strong interest now exists for ATP-competitive inhibitors. The activity of these small molecules differs from the others by blocking both mTORC1-dependent phospholylation of S6K1 and mTORC2-dependent phosphorylation of AKT Ser473 residue. These mTOR inhibitors will probably significantly improve patients' response to $\mathrm{AD}$ therapies [40].

\section{mTOR in pemphigus vulgaris}

Pemphigus vulgaris (PV) is an autoimmune muco-cutaneous disease characterized by intraepidermal blisters and autoantibodies against desmosomal cadherin desmoglein (Dsg) 1 and/or 3 [41].

Other non-desmoglein autoantibodies (acetylcholine receptors, E-cadherin or antimitochondrial autoantibodies), which have also been implicated in the acantholytic process have been detected in the serum of patients with $\mathrm{PV}$ [42].

Different study demonstrated the activation of the apoptosis mechanism in acantholytic PV cells [43].

Pretel $\mathrm{M}$ et al. demostrated that mTOR phosphorylated was highly localized in the basal cells of mice injected with pemphigus vulgaris $\mathrm{IgG}$ and was scattered when the mice were injected with normal human serum. Mice injected with PV IgG and treated with rapamycin showed no signs of suprabasal acantholysis highlighting that mTOR activity contributes to the development of lesions in pemphigus vulgaris $[44,46]$. The imbalance in Akt/mTOR activities caused by PV-IgG within the basal cells may provide the intracellular signals necessary for the development of apoptosis and acantholysis in PV. This Akt/mTOR imbalance may depend on the activation of HER receptor isoforms and the downstream intracellular signalling pathways subsequently triggered in basal cells. Moreover, the activation of HER receptors in basal cells together with the absence of Akt in these cells may explain the restriction of acantholysis to the basal layer of epidermis in PV. These evidences create a new approach to the treatment of pemphigus based on interfering with or blocking the signalling pathways and enzymatic processes that lead to blistering.

\section{mTOR in acne}

Acne is a complex and chronic disorder of the pilosebaceous unit and its pathogenesis is attributed to multiple factors such as hyperseborrhea, hyperkeratinization of the pilosebaceous duct, colonization of Propionibacterium acnes and perifollicular inflammation [47]. In the last years, appeared a clear association between the pathogenesis of acne and the endocrine signalling also influenced by Western diet. Hyperglycaemic diets and insulin tropic foods can increase serum levels of insulin and insulin-like growth factor-1 (IGF-1), predisposing to dysregulation of the sebaceous gland activity triggering hyperseborrhea and to proliferation of acroinfundibular keratinocytes. While a low glycemic, diet seems to improve the clinical manifestations $[48,49]$. Fabbrocini et al. demonstrated that male patients with acne resistant to common therapies might have alterations of metabolic profile and insulin resistance [50]. The same authors showed that in selected cases with an alterated metabolic profile metformin reduces acne lesion [51]. In this context, a decisive role has been attributed to the hyperactivity of the conserved serine/threonine kinase mTORC1.

In 2017 Mastrofrancesco et al. demonstrates that in sebocytes, insulin signalling activated the phosphatidylinositide 3-kinase-Akt (PI3K/Akt) and mammalian target of rapamycin (mTOR) pathways, which, in turn, induced high protein/lipid synthesis, increased cell growth and proliferation as well as inflammation [52]. Tuo et al. have moreover demonstrated in vitro that ALA-PDT suppressed the cell growth in sebocytes cells by mTOR-p70 S6K signalling and reduced the lipogenesis by mTOR-SREBP-1/PPAR $\gamma$ signalling. Sebaceous glands atrophy and reduction of sebum secretion after ALA-PDT may be due to the suppression of lipogenesis and cell growth in sebocytes [53]. Our group has demonstrated mTOR alterations in vivo. mTOR gene expression resulted significantly increased either in NLS or in LS skin from 10 acne patients, respectively, of 17.96- and 20.77-fold (mean values) in comparison of skin biopsies of healthy patients. mTOR gradually increased from HS to LS [54]. This is a preliminary study, with a small number of patients, providing the first evidence that mTOR gene expression is increased and S6K1 is activated in acne patients. It confirms the role of mTORC1 signalling in in the complex inflammatory scenario of acne. Agamia et al. has confirmed that FoxO1, mTOR, serum IGF-1 and a high-glycaemic-load diet may play a role in acne pathogenesis. In fact, they analysed 60 participants, 40 patients with acne and 20 controls [55]. A significantly higher serum IGF-1 level was observed in the patients with acne than in the controls. Skin biopsies in lesional skin have shown the cytoplasmic expression of FoxO1 was significantly greater in the acne group, whereas in the control subjects this expression was likely to be nuclear. Both the cytoplasmic expression and the nuclear expression of mTOR 
were significantly more intense in the patients with acne than in the controls. Moreover, excess consumption of a high-glycaemic-load diet, evaluated trough a diet questionnaire, was significantly associated with higher serum levels of IGF-1 and cytoplasmic expression of FoxO1 and mTOR. Melnik evidences that insulin-IGF-1 signals extrudes FoxO1 into the cytoplasm, whereas nuclear FoxO1 suppresses hepatic IGF-1 synthesis and thus impairs somatic growth. FoxO1 attenuates androgen signalling, interacts with regulatory proteins important for sebaceous lipogenesis, regulates the activity of innate and adaptive immunity, antagonizes oxidative stress and most importantly functions as a rheostat of mTORC1. Thus, FoxO1 links nutrient availability to mTORC1-driven processes: in particular, cell differentiation including hyperproliferation of acroinfundibular keratinocytes, sebaceous gland hyperplasia, increased sebaceous lipogenesis, insulin resistance and increased body mass index. mTORC signal is involved in therapeutic response to acne treatment such as isotretinoin or metformin; in fact, it acts by up regulating FoxO transcription factors [56-59]. Evidence indicates that isotretinoin induces the expression of the transcription factor p53. p53 induces the expression of FoxO1 and PTEN, but inhibits the expression of IGF-1 and androgen receptors, resulting in impaired IGF-1/mTORC1 and androgen signalling [60,61]. All these data confirm that mTOR signalling is involved in the complex pathogenesis of acne and that an high-glycaemic-load diet can be associated with hyper-expression of mTOR. Deeper insights into the molecular interplay of FoxO1/mTORC1-mediated nutrient signalling are thus of critical importance to understand the impact of western diet on acne and to evaluate therapeutic response to acne treatment.

\section{mTOR and HS}

HS is a chronic, inflammatory, recurrent, debilitating, follicular skin disease that usually presents after puberty with painful deep seated, inflamed lesions in the apocrine gland-bearing areas of the body. Acne and hidradenitis suppurativa (HS) are both pathologies of the hair follicles and innate inflammation has been considered the central driving factor to both diseases [62].

As mentioned in previous paragraph mTOR dysregulation is involved in different inflammatory conditions and is altered in metabolic and in autoimmune disorders. Recently, Monfrecola et al. have for the first time investigated the association between mTOR and HS. They found mTOR increased in lesional as well as non-lesional skin of HS patients. Moreover, mTOR gene expression statistically correlated with the severity of HS. In this study it was moreover investigated the association between mTOR and insulin resistance. Furthermore, insulin resistance HS subjects presented also an intense correlation between serum insulin during OGTT at 30 as well as $60 \mathrm{~min}$ and mTOR gene expression. They suggested that mTOR might represent molecular marker related to HS and could be indicative of associated insulin resistance. Furthermore, it was observed that mTORC1 signalling might be modulated by anti TNFa therapy [63]. Recently, in addition to the link between HS, metabolic syndrome and mTOR, the focus has been on the role of mTOR in the immune system regulation and in the pathogenesis of HS. In fact, mTOR pathway has a fundamental role in the differentiation of Th17 cells that play a central function in HS pathogenesis $[64,65]$.

Moreover, alterated activation and proliferation of macrophages and secretion of pro-inflammatory cytokines acts a crucial role in HS pathogenesis. In particular, macrophages are the most numerous inflammatory cells found in HS infiltrate and release numerous proinflammatory cytokines such as interleukin (IL)-23, IL-1b and tumour necrosis factor (TNF)-alpha. Particularly mTORC1 signaling seems to have a crucial role in the control of macrophage metabolism and activation. In fact, according to some authors, alteration of mTORC can be related to macrophage dysregulation in HS $[65,66]$. mTORC1mediated increase in protein synthesis supports the production of high levels of pro-inflammatory cytokines, chemokines and other factors in activated macrophages. All these data suggest that mTOR might represent a molecular marker related to severity of $\mathrm{HS}$ and indicative of associated insulin resistance, obesity and immune system alteration but also suggests a possible mechanism of action of some treatment such as metformin and isotretinoin in HS [67].

\section{Therapeutic implications}

Deeper insights into mTOR regulation and signalling may help dermatologists to better understand the central role of the Western diet in the pathogenesis of different inflammatory skin diseases. These findings could be sufficient to recommend a nutritional therapy for mTOR-related skin disorders: normalize total calorie intake, lower glycaemic load and restrict total dairy protein consumption, especially whey protein abuse.

\section{Conclusion}

mTOR pathway is involved in the pathogenesis of several dermatological conditions. All the evidences analyzed in this review supports the idea that mTOR pathway plays an important role in inflammatory and proliferative skin diseases related to metabolic alteration. Further study will be necessary to evaluate the potential role of mTOR as biological marker of glycemic metabolic impairment and its modification as therapeutic target in different skin disease.

\section{Funding souraces}

None declared

\section{Conflicts of interest}

None of the contributing authors have any conflict of interest, including specific financial interests of relationships and affiliation relevant to the subject matter or discussed materials in the manuscript.

\section{References}

1. Brown EJ, Albers MW, Shin TB, Ichikawa K, Keith CT, et al. (1994) A mammalian protein targeted by G1-arresting rapamycin-receptor complex. Nature 369: 756-758. [Crossref]

2. Kim LC, Cook RS, Chen J (2017) mTORC1 and mTORC2 in cancer and the tumor microenvironment. Oncogene 36: 2191-2201. [Crossref]

3. Alessi DR, Andjelkovic M, Caudwell B, Cron P, Morrice N, et al. (1996) Mechanism of activation of protein kinase B by insulin and IGF-1. EMBO J 15: 6541-6551. [Crossref]

4. Alessi DR, James SR, Downes CP, Holmes AB, Gaffney PR, et al. (1997) Characterization of a 3-phosphoinositidedependent protein kinase which phosphorylates and activates protein kinase Balpha. Curr Biol 7: 261-269. [Crossref]

5. Deng Y, Yang J, Luo F, Qian J, Liu R, et al. (2018) mTOR-mediated glycolysis contributes to the enhanced suppressive function of murine tumor-infiltrating monocytic myeloid-derived suppressor cells. Cancer Immunol Immunother 9: 13551364. [Crossref]

6. Delgoffe GM, Pollizzi KN, Waickman AT, Heikamp E, Meyers DJ, et al. (2011) The kinase mTOR regulates the differentiation of helper $\mathrm{T}$ cells through the selective activation of signaling by mTORC1 and mTORC2. Nat Immunol 12: 295-303. [Crossref]

7. Long SH, He Y, Chen MH, Cao K, Chen YJ, et al. (2013) Activation of PI3K/Akt mTOR signaling pathway triggered by PTEN downregulation in the pathogenesis of Crohn's disease. J Dig Dis 14: 662-669. [Crossref]

8. Syed DN, Afaq F, Mukhtar H (2012) Differential activation of signaling pathways by UVA and UVB radiation in normal human epidermal keratinocytes. Photochem Photobiol 88: 1184-1190. [Crossref] 
9. Karbowniczek M, Spittle CS, Morrison T, Wu H, Henske EP (2008) mTOR is activated in the majority of malignant melanomas. J Invest Dermatol 128: 980-987. [Crossref]

10. Matsui T, Amagai M (2015) Dissecting the formation, structure and barrier function of the stratum corneum. Int Immunol 27: 269-280. [Crossref]

11. Buerger C, Shirsath N, Lang V, Berard A, Diehl S, et al. (2017) Inflammation dependen mTORC1 signaling interferes with the switch from keratinocyte proliferation to differentiation. PLoS One 12: e0180853. [Crossref]

12. Datta Mitra A, Raychaudhuri SP, Abria CJ, Mitra A, Wright R, et al. (2013) 1a,25Dihydroxyvitamin-D3-3 bromoacetate regulates AKT/mTOR signaling cascades: a therapeutic agent for psoriasis. J Invest Dermatol 133: 1556-1564. [Crossref]

13. Lowes MA, Russell CB, Martin DA, Towne JE, Krueger JG (2013) The IL-23/T17 pathogenic axis in psoriasis is amplified by keratinocyte responses. Trends Immunol 34: 174-181. [Crossref]

14. Boehncke WH (2015) Etiology and Pathogenesis of Psoriasis. Rheum Dis Clin North Am 41: 665-675. [Crossref]

15. Ding X, Bloch W, Iden S, Rüegg MA, Hall MN, et al. (2016) mTORC1 and mTORC2 regulate skin morphogenesis and epidermal barrier formation. Nat Commun 7: 13226. [Crossref]

16. Buerger C, Malisiewicz B, Eiser A, Hardt K, Boehncke WH (2013) Mammalian target of rapamycin and its downstream signaling components are activated in psoriatic skin. Br J Dermatol 169: 156-159. [Crossref]

17. Burger C, Shirsath N, Lang V, Diehl S, Kaufmann R, et al. (2017) Blocking mTORSignalling with Rapamycin Ameliorates Imiquimod-induced Psoriasis in Mice. Acta Derm Venereol 97: 1087-1094.

18. Huang T, Lin X, Meng X, Lin M (2014) Phosphoinositide-3 kinase/ protein kinase-B/ mammalian target of rapamycin pathway in psoriasis pathogenesis. A potential therapeutic target? Acta DermVenereol 94: 371-379. [Crossref]

19. Inoki K, Ouyang H, Li Y, Guan KL (2007) Signaling by target of rapamycin proteins in cell growth control. Microbiol Mol Biol Rev 69: 79-100. [Crossref]

20. Lee CH, Inoki K, Guan KL (2007) mTOR pathway as a target in tissue hypertrophy. Annu Rev Pharmacol Toxicol 47: 443-467. [Crossref]

21. Raychaudhuri SK, Raychaudhuri SP (2014) mTOR Signaling Cascade in Psoriatic Disease: Double Kinase mTOR Inhibitor a Novel Therapeutic Target. Indian $J$ Dermatol 59: 67-70. [Crossref]

22. Mitra D, Raychaudhuri SP, Abria CJ, Mitra A, Wright R, et al. (2013) 1a,25Dihydroxyvitamin-D3-3-Bromoacetate Regulates AKT/mTORSignaling Cascades: A Therapeutic Agent for Psoriasis. J Invest Dermatol 133: 1556-1564.

23. Tiberio R, Bozzo C, Pertusi G, Graziola F, Gattoni M, et al. (2009) Calcipotriol induces apoptosis in psoriatic keratinocytes. Clin Exp Dermatol 34: e972-974. [Crossref]

24. Balato A, Lembo S, Ayala F, Balato N, Caiazzo G, et al. (2017) Mechanistic target of rapamycin complex 1 is involved in psoriasis and regulated by anti-TNF- $\hat{I} \pm$ treatment. Exp Dermatol 26: 325-327. [Crossref]

25. Leo MS, Sivamani RK (2014) Phytochemical modulation of the Akt/mTOR pathway and its potential use in cutaneous disease. Arch Dermatol Res 306: 861-871. [Crossref]

26. Balato A, Di Caprio R, Lembo S, Mattii M, Megna M, et al. (2014) Mammalian target of rapamycin in inflammatory skin conditions. Eur J Inflamm 12: 341-350.

27. Lee CH, Chuang HY, Shih CC, Jong SB, Chang CH, et al. (2006) Transepidermal water loss, serum IgE and beta-endorphin as important and independent biological markers for development of itch intensity in atopic dermatitis. Br J Dermatol 154: 1100-1107. [Crossref]

28. Furue M (1994) Atopic dermatitis--immunological abnormality and its background. $J$ Dermatol Sci 7: 159-168.

29. Tollefson MM, Bruckner AL; Section on Dermatology (2014) Atopic dermatitis: skindirected management. Pediatrics 134: e1735-1744. [Crossref]

30. Siraganian RP (2003) Mast cell signal transduction from the high-affinity IgE receptor. Curr Opin Immunol 15: 639-646. [Crossref]

31. Luskova P, Draber P (2004) Modulation of the Fcepsilon receptor I signaling by tyrosine kinase inhibitors: search for therapeutic targets of inflammatory and allergy diseases. Curr Pharm Des 10: 1727-1737. [Crossref]

32. Smrz D, Kim MS, Zhang S, Mock BA, Smrzova S, et al. (2011) mTORC1 and mTORC2 differentially regulate homeostasis of neoplastic and non-neoplastic human mast cells. Blood 118: 6803-6813. [Crossref]
33. Yang F, Tanaka M, Wataya-Kaneda M, Yang L, Nakamura A, et al. (2014) Topical application of rapamycin ointment ameliorates Dermatophagoides farina body extractinduced atopic dermatitis in NC/Nga mice. Exp Dermatol 23: 568-572. [Crossref]

34. Osada-Oka M, Hirai S, Izumi Y, Misumi K, Samukawa K, et al. (2018) Red ginseng extracts attenuate skin inflammation in atopic dermatitis through p70 ribosomal protein S6 kinase activation. $J$ Pharmacol Sci 136: 9-15. [Crossref]

35. Nguyen CT, Luong TT, Kim GL, Pyo S, Rhee DK (2015) Korean red ginseng inhibits apoptosis in neuroblastoma cells via estrogen receptor b-mediated phosphatidylinositol-3 kinase/Akt signaling. J Ginseng Res 39: 69e75.

36. Osada-Oka M, Hirai S, Izumi Y, Misumi K, Samukawa K3, et al. (2018) Red ginseng extracts attenuate skin inflammation in atopic dermatitis through $\mathrm{p} 70$ ribosomal protein S6 kinase activation. J Pharmacol Sci 136: 9-15. [Crossref]

37. Rebane A, Zimmermann M, Aab A, Baurecht H, Koreck A, et al. (2012) Mechanisms of IFN- $\hat{I}^{3}$-induced apoptosis of human skin keratinocytes in patients with atopic dermatitis. J Allergy Clin Immunol 129: 1297-1306. [Crossref]

38. Ma L, Xue HB, Guan XH, Shu CM, Wang F, et al. (2014) The Imbalance of Th17 cells and CD4(+) CD25(high) Foxp3(+) Treg cells in patients with atopic dermatitis. $J$ Eur Acad Dermatol Venereol 28: 1079-1086. [Crossref]

39. Bae MJ, Lim S, Lee DS, Ko KR, Lee W, et al. (2016) Water-soluble extracts from Actinidia arguta, PG102, attenuates house dust mite-induced murineatopic dermatitis by inhibiting the mTOR pathway with Treg generation. J Ethnopharmacol 193: 96-106. [Crossref]

40. Ding X, Aoki V, Mascaro JM Jr, Lopez-Swiderski A, Diaz LA, et al. (1997) Mucosa and mucocutaneous (general-ized) pemphigus vulgaris show distinct autoantibody profiles. J Invest Dermatol 109: 592-596. [Crossref]

41. Hamilton JD, Suárez-Fariñas M, Dhingra N, Cardinale I, Li X, et al. (2014) Dupilumab improves the molecular signature in skin of patients with moderate-to-severe atopic dermatitis. J Allergy Clin Immunol 134: 1293-1300. [Crossref]

42. Zhang YJ, Duan Y, Zheng XF (2011) Targeting the mTOR kinase domain: the second generation of mTOR inhibitors. Drug Discov Today 16: 325-331. [Crossref]

43. Taylor RC, Cullen SP, Martin SJ (2008) Apoptosis: controlled demolition at the cellular level. Nat Rev Mol Cell Biol 9: 231-241. [Crossref]

44. Calautti E, Li J, Saoncella S, Brissette JL, Goetinck PF (2005) Phosphoinositide 3-kinase signaling to Akt promotes keratinocyte differentiation versus death. $J$ BiolChem 280: 32856-32865. [Crossref]

45. Pretel M, España A, Marquina M, Pelacho B, López-Picazo JM, et al. (2009) An imbalance in Akt $/ \mathrm{mTOR}$ is involved in the apoptotic and acantholytic processes in a mouse model of pemphigus vulgaris. Exp Dermatol 18: 771-780. [Crossref]

46. Grando SA, Bystryn JC, Chernyavsky AI, Frusić-Zlotkin M, Gniadecki R, et al. (2009) Apoptolysis: a novel mechanism of skin blistering in pemphigus vulgaris linking the apoptotic pathways to basal cell shrinkage and suprabasalacantholysis. Exp Dermatol 18: 764-770. [Crossref]

47. Fabbrocini G, Annunziata MC, D'Arco V, De Vita V, Lodi G, et al. (2010) Acne scars: pathogenesis, classification and treatment. Dermatol Res Pract 2010: 893080. [Crossref]

48. Kim DH, Sarbassov DD, Ali SM, King JE, Latek RR, et al. (2002) European Journal of Inflammation $349 \mathrm{DM}$. mTOR interacts with raptor to form a nutrientsensitive complex that signals to the cell growth machinery. Cell 110: 163-167. [Crossref]

49. Del Prete M, Mauriello MC, Faggiano A, Di Somma C, Monfrecola G, et al. (2012) Insulin resistance and acne: a new risk factor for men? Endocrine 42: 555-560. [Crossref]

50. Fabbrocini G, Izzo R, Faggiano A, Del Prete M, Donnarumma M, et al. (2016) Low glycaemic diet and metformin therapy: a new approach in male subjects with acne resistant to common treatments. Clin Exp Dermatol 41: 38-42. [Crossref]

51. Mastrofrancesco A, Ottaviani M, Cardinali G, Flori E, Briganti S, et al. (2017) Pharmacological PPAR $\hat{I}^{3}$ modulation regulates sebogenesis and inflammation in SZ95 human sebocytes. Biochem Pharmacol 138: 96-106. [Crossref]

52. Tuo J, Wang Q, Zouboulis CC, Liu Y, Ma Y, et al. (2017) ALA-PDT suppressing the cell growth and reducing the lipogenesis in human SZ95 sebocytes by mTOR signaling pathway in vitro. Photodiagnosis Photodyn Ther 18: 295-301. [Crossref]

53. Monfrecola G, Lembo S, Caiazzo G, De Vita V, Di Caprio R, et al. (2016) Mechanistic target of rapamycin (mTOR) expression is increased in acne patients' skin. Exp Dermatol 25: 153-155. [Crossref] 
54. Agamia NF, Abdallah DM, Sorour O, Mourad B, Younan DN, et al. (2016) Skin expression of mammalian target of rapamycin and forkhead box transcription factor O1, and serum insulin-like growth factor-1 in patients with acne vulgaris and their relationship with diet. Br J Dermatol 174: 1299-1307

55. Melnik BC (2010) Is nuclear deficiency of FoxO1 due to increased growth factor/PI3K Akt-signalling in acne vulgaris reversed by isotretinoin treatment? Br J Dermatol 162 : 1398-1400. [Crossref]

56. Melnik BC (2010) The role of transcription factor FoxO1 in the pathogenesis of acne vulgaris and the mode of isotretinoin action. G Ital Dermatol Venereol 145: 559-571. [Crossref]

57. Melnik BC (2011) Isotretinoin and FoxO1: A scientific hypothesis. Dermatoendocrinol 3 : 141-165. [Crossref]

58. Melnik BC, Schmitz G (2013) Are therapeutic effects of antiacne agents mediated by activation of FoxO1 and inhibition of mTORC1? Exp Dermatol 22: 502-504. [Crossref]

59. Melnik BC (2017) Apoptosis May Explain the Pharmacological Mode of Action and Adverse Effects of Isotretinoin, Including Teratogenicity. Acta Derm Venereol 97: 173181. [Crossref]

60. Melnik BC (2017) p53: key conductor of all anti-acne therapies. J Transl Med 15: 195. [Crossref]
61. Pink A, Anzengruber F, Navarini AA (2018) Acne and hidradenitis suppurativa. $B r J$ Dermatol 178: 619-631. [Crossref]

62. Balato A, Caiazzo G, Annunziata MC, Marasca C, Scala E, et al. (2019) Anti-TNFa therapy modulates mTORC1signalling in hidradenitis suppurativa. J Eur Acad Dermatol Venereol. 33: e43-e45. [Crossref]

63. Nagai S, Kurebayashi Y, Koyasu S (2013) Role of PI3K/Akt and mTOR complexes in Th17 cell differentiation. Ann N Y Acad Sci 1280: 30-34. [Crossref]

64. Ren W, Yin J, Duan J, Liu G, Tan B, et al. (2016) mTORC1 signaling and IL-17 expression: defining pathways and possible therapeutic targets. Eur J Immunol 46: 291299. [Crossref]

65. Moran B, Sweeney CM, Hughes R, Malara A, Kirthi S, et al. (2017) Hidradenitis Suppurativa Is Characterized by Dysregulation of the Th17: Treg Cell Axis, Which Is Corrected by Anti-TNF Therapy. J Invest Dermatol 137: 2389-2395. [Crossref]

66. Marasca C, Balato A, Annunziata MC, Cacciapuoti S, Fabbrocini G (2018) Insulin resistance, mTOR and hidradenitis suppurativa. J Eur Acad Dermatol Venereol. [Crossref]

67. Monfrecola G, Balato A, Caiazzo G, De Vita V, Di Caprio R, et al. (2016) Mammalian target of rapamycin, insulin resistance and hidradenitis suppurativa: a possible metabolic loop. J Eur Acad Dermatol Venereol 30: 1631-1633. [Crossref]

Copyright: (2019 Cacciapuoti S. This is an open-access article distributed under the terms of the Creative Commons Attribution License, which permits unrestricted use, distribution, and reproduction in any medium, provided the original author and source are credited. 